University of Nebraska - Lincoln

DigitalCommons@University of Nebraska - Lincoln

\title{
Electron-Impact Ionization Cross Sections of Atmospheric Molecules
}

\author{
Y.-K. Kim \\ National Institute of Standards and Technology, Gaithersburg, Maryland \\ W. Hwang \\ National Institute of Standards and Technology, Gaithersburg, Maryland \\ N. M. Weinberger \\ National Institute of Standards and Technology, Gaithersburg, Maryland
}

M. A. Ali

Howard University, Washington, D.C.

M. Eugene Rudd

University of Nebraska - Lincoln, erudd@unl.edu

Follow this and additional works at: https://digitalcommons.unl.edu/physicsrudd

Part of the Physics Commons

Kim, Y.-K.; Hwang, W.; Weinberger, N. M.; Ali, M. A.; and Rudd, M. Eugene, "Electron-Impact lonization Cross Sections of Atmospheric Molecules" (1997). M. Eugene Rudd Publications. 20.

https://digitalcommons.unl.edu/physicsrudd/20

This Article is brought to you for free and open access by the Research Papers in Physics and Astronomy at DigitalCommons@University of Nebraska - Lincoln. It has been accepted for inclusion in M. Eugene Rudd Publications by an authorized administrator of DigitalCommons@University of Nebraska - Lincoln. 


\title{
Electron-impact ionization cross sections of atmospheric molecules
}

\section{J. Chem. Phys. 106, 1026 (1997); DOI:10.1063/1.473186}

Issue Date: 15 January 1997

Y.-K. Kim, W. Hwang, and N. M. Weinberger

National Institute of Standards and Technology, Gaithersburg, Maryland 20899

M. A. Ali

Department of Chemistry, Howard University, Washington, D.C. 20059

M. E. Rudd

Department of Physics and Astronomy, University of Nebraska-Lincoln, Lincoln, Nebraska 68588-0111

\begin{abstract}
A theoretical model for electron-impact total ionization cross sections, which has been found to be reliable for a wide range of molecules, is applied to molecules of interest to atmospheric science. The new theory, the binary-encounter-Bethe (BEB) model, combines the binaryencounter theory and the Bethe theory for electron-impact ionization, and uses simple theoretical data for the ground state of the target molecule, which are readily available from molecular structure codes. Total ionization cross sections of 11 molecules, $\mathrm{CS}, \mathrm{CS}_{2}, \mathrm{COS}, \mathrm{CH}_{4}, \mathrm{H}_{2} \mathrm{~S}, \mathrm{NH}_{3}$, $\mathrm{NO}_{2}, \mathrm{~N}_{2} \mathrm{O}, \mathrm{O}_{3}, \mathrm{~S}_{2}$, and $\mathrm{SO}_{2}$, are presented for incident electron energies from threshold to $1 \mathrm{keV}$ with an average accuracy of $15 \%$ or better at the cross section peak. We also found that the use of vertical ionization potentials (IPs) rather than adiabatic IPs for the lowest IPs significantly improves BEB cross sections between the threshold and cross section peak for molecules whose adiabatic and vertical IPs are different by $\sim 1 \mathrm{eV}$ or more $\left(\mathrm{CH}_{4}\right.$ and $\left.\mathrm{NH}_{3}\right)$. The BEB cross sections are presented in a compact analytic form with a small number of constants, making the cross sections suitable for modeling applications.

(C)1997 American Institute of Physics.
\end{abstract}

History: $\quad$ Received 20 August 1996; accepted 11 October 1996

Permalink: http://link.aip.org/link/?JCPSA6/106/1026/1 


\title{
Electron-impact ionization cross sections of atmospheric molecules
}

\author{
Y.-K. Kim, W. Hwang, ${ }^{\text {a) }}$ and N. M. Weinberger ${ }^{\text {b) }}$ \\ National Institute of Standards and Technology, Gaithersburg, Maryland 20899 \\ M. A. Ali \\ Department of Chemistry, Howard University, Washington, D.C. 20059 \\ M. E. Rudd \\ Department of Physics and Astronomy, University of Nebraska-Lincoln, Lincoln, Nebraska 68588-0111
}

(Received 20 August 1996; accepted 11 October 1996)

\begin{abstract}
A theoretical model for electron-impact total ionization cross sections, which has been found to be reliable for a wide range of molecules, is applied to molecules of interest to atmospheric science. The new theory, the binary-encounter-Bethe (BEB) model, combines the binary-encounter theory and the Bethe theory for electron-impact ionization, and uses simple theoretical data for the ground state of the target molecule, which are readily available from molecular structure codes. Total ionization cross sections of 11 molecules, $\mathrm{CS}, \mathrm{CS}_{2}, \mathrm{COS}, \mathrm{CH}_{4}, \mathrm{H}_{2} \mathrm{~S}, \mathrm{NH}_{3}, \mathrm{NO}_{2}, \mathrm{~N}_{2} \mathrm{O}, \mathrm{O}_{3}, \mathrm{~S}_{2}$, and $\mathrm{SO}_{2}$, are presented for incident electron energies from threshold to $1 \mathrm{keV}$ with an average accuracy of $15 \%$ or better at the cross section peak. We also found that the use of vertical ionization potentials (IPs) rather than adiabatic IPs for the lowest IPs significantly improves BEB cross sections between the threshold and cross section peak for molecules whose adiabatic and vertical IPs are different by $\sim 1 \mathrm{eV}$ or more $\left(\mathrm{CH}_{4}\right.$ and $\left.\mathrm{NH}_{3}\right)$. The BEB cross sections are presented in a compact analytic form with a small number of constants, making the cross sections suitable for modeling applications. (C) 1997 American Institute of Physics. [S0021-9606(97)01703-0]
\end{abstract}

\section{INTRODUCTION}

In earlier publications, ${ }^{1,2}$ we have demonstrated that a new theoretical method, the binary-encounter-Bethe (BEB) model, provides reliable electron-impact total ionization cross sections for molecules using very simple input data for the ground state, all of which can be obtained from standard molecular wave function codes. There are no adjustable or fitted parameters in the theory. The BEB model does not provide details such as resonances in the continuum, vibrational and/or rotational excitations concomitant with ionization, multiple ionization, and dissociative ionization. It simply predicts the total ionization cross section as the sum of ionization cross sections for ejecting one electron from each of the molecular orbitals. We also have demonstrated that it is valid from the first ionization threshold to several $\mathrm{keV}$ incident electron energies.

In this article, we apply the BEB model to 11 molecules of interest to atmospheric science: $\mathrm{CS}, \mathrm{CS}_{2}, \mathrm{COS}, \mathrm{CH}_{4}$, $\mathrm{H}_{2} \mathrm{~S}, \mathrm{NH}_{3}, \mathrm{NO}_{2}, \mathrm{~N}_{2} \mathrm{O}, \mathrm{O}_{3}, \mathrm{~S}_{2}$, and $\mathrm{SO}_{2}$. Some of these molecules, e.g., $\mathrm{H}_{2} \mathrm{~S}$, are not only pollutants on the Earth but have been found on comets. ${ }^{3}$ As was the case for about two dozen atoms and molecules reported earlier, ${ }^{1,2}$ the BEB model provides cross sections in good agreement with available experimental data. To the best of our knowledge, our total ionization cross section for $\mathrm{NO}_{2}$ is the first of its kind reported in the literature.

In earlier applications of the BEB model, we chose to

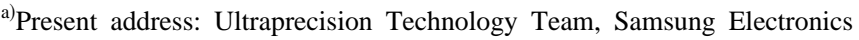
Co., Suwon, Kyonggi-do, Korea.

${ }^{b}$ Present address: Dept. of Chemistry, Univ. of California, Irvine, California 92715.
}

use experimental adiabatic ionization potentials (IPs) for the lowest IP so that the BEB cross section will have appropriate threshold to compare to experimental data. We used adiabatic IPs for this purpose, mainly because this type of IPs are easy to find for many molecules and radicals. However, adiabatic IPs are not associated with a specific molecular orbital but with the appearance of specific ion products, while vertical IPs are associated with individual molecular orbitals in the ground state of the neutral molecule. Since the BEB model is based on molecular orbitals, it seems more appropriate to use the vertical IPs, as is done in photoionization. Although the BEB cross section is insensitive to a small shift in an IP value, we found that using vertical IPs significantly improved the agreement between theory and experiment when the adiabatic and vertical IPs are different by $\sim 1 \mathrm{eV}$ or more. Relatively large differences in adiabatic and vertical IPs are observed in molecules whose geometrical structure changes drastically when ionized or in molecules subject to strong Jahn-Teller effect, i.e., molecules with valence orbitals of high symmetry. When we used vertical IPs, the BEB cross sections for $\mathrm{CH}_{4}$ and $\mathrm{NH}_{3}$ improved considerably near the threshold compared to available experiments.

The main advantages of the BEB model are twofold: the theory is valid for the entire incident energy range from threshold to several $\mathrm{keV}$, and it is in an analytic form with simple constants providing compact analytic expressions for the total ionization cross section suitable for applications in atmospheric modeling.

The underlying theory is briefly outlined in Sec. II, the total ionization cross sections of individual molecules are presented in Sec. III, and conclusions are presented in Sec. IV. 


\section{OUTLINE OF THEORY}

The BEB model is a simpler version of the binaryencounter-Dipole (BED) model for electron-impact ionization cross sections of atoms and molecules. ${ }^{2}$ The BED model combines the binary-encounter theory ${ }^{4}$ and the Bethe theory. ${ }^{5}$ The connection between the binary-encounter theory and the Bethe theory is determined by requiring the asymptotic form at high incident energy $T$ of the former to match that of the latter both in the ionization cross section and in the stopping cross section. The stopping cross section is used to evaluate the stopping power of the target medium. The BED model provides a formula to calculate the singly differential cross section, or the energy distribution of ejected electrons $d \sigma / d W$ with the ejected electron energy $W$, for each atomic or molecular orbital. To apply the BED model, one needs for each orbital the electron binding energy $B$, the average kinetic energy $U=\left\langle\mathbf{p}^{2} / 2 m\right\rangle$ with the bound electron momentum $\mathbf{p}$ and the electron mass $m$, the electron occupation number $N$ and the continuum dipole oscillator strength $d f / d W$.

The value of the kinetic energy $U$ for each orbital in the initial state (usually the ground state) of the target is a theoretical quantity evaluated in any atomic or molecular wave function code that calculates the total energy. However, both the initial- and continuum-state wave functions are needed to calculate $d f / d W$ and this is the only nontrivial data needed to apply the BED model. Alternatively, $d f / d W$ can be deduced from experimental photoionization cross sections, though partial cross sections are needed to deduce $d f / d W$ for each orbital.

For most molecules, however, it is difficult in practice to obtain $d f / d W$ for each orbital. To alleviate this difficulty, $d f / d W$ is approximated in the BEB model by choosing

$$
\frac{d f}{d W} \propto(B+W)^{-2} .
$$

This form of $d f / d W$ closely resembles the shape of the $d f / d W$ of the hydrogen atom in the ground state.

With this simplified $d f / d W$, the integrated ionization cross section per molecular orbital, $\sigma_{\mathrm{BEB}}$, is obtained by integrating $d \sigma / d W$ from $W=0$ to $(T-B) / 2$. The result is

$$
\sigma_{\mathrm{BEB}}=\frac{S}{t+u+1}\left[\frac{\ln t}{2}\left(1-\frac{1}{t^{2}}\right)+1-\frac{1}{t}-\frac{\ln t}{t+1}\right],
$$

where $t=T / B, u=U / B, S=4 \pi a_{0}^{2} N R^{2} / B^{2}, a_{0}=5.292 \AA$, and $R=13.61 \mathrm{eV}$.

In Eq. (2), the term associated with the first logarithmic function on the right-hand side (rhs) represents distant collisions (large impact parameters) dominated by the dipole interaction, and the rest of the terms on the rhs represent close collisions (small impact parameters) as described by the Mott cross section. ${ }^{6}$ The second logarithmic function originates from the interference of the direct and exchange scattering also described by the Mott cross section.

We present the values of $B, U$ and $N$ for the 11 molecules in this article in Tables I and II. The molecular wave function data are from the molecular structure code
TABLE I. Molecular orbitals, electron binding energy $B$ in eV, kinetic energy $U$ in $\mathrm{eV}$, and electron occupation number $N$ for $\mathrm{CS}, \mathrm{CS}_{2}, \mathrm{COS}$, and $\mathrm{H}_{2} \mathrm{~S}$. All $B$ and $U$ values are theoretical, except that those marked by an asterisk are experimental adiabatic IPs, and those marked by a dagger are

\begin{tabular}{|c|c|c|c|c|}
\hline Molecule & MO & B & $\mathrm{U}$ & $\mathrm{N}$ \\
\hline \multirow[t]{8}{*}{$\mathrm{CS}$} & $2 \sigma$ & 308.84 & 436.58 & 2 \\
\hline & $3 \sigma$ & 245.09 & 509.11 & 2 \\
\hline & $4 \sigma$ & 182.00 & 478.18 & 2 \\
\hline & $1 \pi$ & 181.94 & 478.41 & 4 \\
\hline & $5 \sigma$ & 30.24 & 59.97 & 2 \\
\hline & $6 \sigma$ & 18.75 & 58.81 & 2 \\
\hline & $7 \sigma$ & 12.76 & 44.37 & 2 \\
\hline & $2 \pi$ & $11.33^{*}$ & 41.13 & 4 \\
\hline \multirow[t]{13}{*}{$\mathrm{CS}_{2}$} & $2 \sigma_{g}$ & 310.28 & 436.25 & 2 \\
\hline & $2 \sigma_{u}$ & 244.77 & 509.19 & 2 \\
\hline & $3 \sigma_{g}$ & 244.77 & 509.21 & 2 \\
\hline & $3 \sigma_{u}^{s}$ & 181.71 & 478.04 & 2 \\
\hline & $4 \sigma_{g}$ & 181.71 & 478.08 & 2 \\
\hline & $1 \pi_{g}^{\delta}$ & 181.62 & 478.73 & 4 \\
\hline & $1 \pi_{u}^{\delta}$ & 181.62 & 478.73 & 4 \\
\hline & $5 \sigma_{g}$ & 31.38 & 56.26 & 2 \\
\hline & $4 \sigma_{u}^{\delta}$ & 27.97 & 63.47 & 2 \\
\hline & $6 \sigma_{g}$ & 18.43 & 63.21 & 2 \\
\hline & $5 \sigma_{u}^{8}$ & 15.88 & 58.27 & 2 \\
\hline & $2 \pi_{u}$ & 14.44 & 37.86 & 4 \\
\hline & $2 \pi_{g}(\mathrm{~S} 3 \mathrm{p})$ & $10.07^{*}$ & $48.07 / 3$ & 4 \\
\hline \multirow[t]{11}{*}{ COS } & $2 \sigma$ & 562.30 & 794.47 & 2 \\
\hline & $3 \sigma$ & 311.20 & 436.01 & 2 \\
\hline & $4 \sigma$ & 244.57 & 509.20 & 2 \\
\hline & $5 \sigma$ & 181.52 & 477.84 & 2 \\
\hline & $1 \pi$ & 181.41 & 478.77 & 4 \\
\hline & $6 \sigma$ & 41.95 & 77.75 & 2 \\
\hline & $7 \sigma$ & 29.58 & 59.03 & 2 \\
\hline & $8 \sigma$ & 21.53 & 73.79 & 2 \\
\hline & $2 \pi$ & 18.48 & 51.09 & 4 \\
\hline & $9 \sigma$ & 17.21 & 60.71 & 2 \\
\hline & $3 \pi$ & $11.17^{*}$ & 48.83 & 4 \\
\hline \multirow[t]{3}{*}{$\mathrm{CH}_{4}$} & $1 a_{1}$ & 290.70 & 436.07 & 2 \\
\hline & $2 \mathrm{a}_{1}$ & 25.73 & 33.05 & 2 \\
\hline & $1 t_{2}$ & $14.25^{\dagger}$ & 25.96 & 6 \\
\hline \multirow[t]{8}{*}{$\mathrm{H}_{2} \mathrm{~S}$} & $2 a_{1}$ & 244.06 & 509.15 & 2 \\
\hline & $1 b_{1}$ & 181.00 & 477.97 & 2 \\
\hline & $3 a_{1}$ & 180.96 & 478.37 & 2 \\
\hline & $1 b_{2}$ & 180.89 & 479.07 & 2 \\
\hline & $4 a_{1}$ & 26.85 & 55.39 & 2 \\
\hline & $2 b_{1}$ & 16.34 & 35.77 & 2 \\
\hline & $5 a_{1}$ & 13.54 & 46.09 & 2 \\
\hline & $2 b_{2}(\mathrm{~S} 3 \mathrm{p})$ & $10.48^{\dagger}$ & $45.68 / 3$ & 2 \\
\hline \multirow[t]{4}{*}{$\mathrm{NH}_{3}$} & $1 a_{1}$ & 422.61 & 601.69 & 2 \\
\hline & $2 a_{1}$ & 31.13 & 48.49 & 2 \\
\hline & $1 \mathrm{e}$ & 17.19 & 35.62 & 4 \\
\hline & $3 a_{1}$ & $10.85^{\dagger}$ & 43.25 & 2 \\
\hline
\end{tabular}
experimental vertical IPs.

GAMESS. ${ }^{7}$ Since deep inner shells, such as the K shell of S, contribute little to the total ionization cross sections, we have omitted them from the tables. One can use either theoretical or experimental values of $B$, while $U$ is a theoretical quantity that cannot be directly measured, though the sum of all $U$ 's is equal to the magnitude of the total energy of the target molecule according to the virial theorem. Since experimental 
TABLE II. Molecular orbitals, electron binding energy $B$ in $\mathrm{eV}$, kinetic energy $U$ in $\mathrm{eV}$, and electron occupation number $N$ for $\mathrm{NO}_{2}, \mathrm{~N}_{2} \mathrm{O}, \mathrm{O}_{3}$, $\mathrm{S}_{2}$, and $\mathrm{SO}_{2}$. All $B$ and $U$ values are theoretical, except that those marked by an asterisk are experimental adiabatic IPs, and those marked by a dagger are experimental vertical IPs.

\begin{tabular}{|c|c|c|c|c|}
\hline Molecule & MO & B & $\mathrm{U}$ & $\mathrm{N}$ \\
\hline \multirow{12}{*}{$\begin{array}{c}\mathrm{NO}_{2} \text {, doublet } \\
\text { average of } \alpha \text { and } \beta \text { orbital values }\end{array}$} & $1 b_{1}$ & 562.48 & 794.45 & 2 \\
\hline & $1 a_{1}$ & 562.48 & 794.46 & 2 \\
\hline & $2 a_{1}$ & 431.59 & 602.08 & 2 \\
\hline & $3 a_{1}$ & 46.11 & 73.41 & 2 \\
\hline & $2 b_{1}$ & 41.06 & 79.40 & 2 \\
\hline & $4 a_{1}$ & 25.00 & 78.97 & 2 \\
\hline & $5 a_{1}$ & 21.60 & 56.73 & 2 \\
\hline & $3 b_{1}$ & 21.46 & 73.27 & 2 \\
\hline & $1 b_{2}$ & 21.19 & 51.57 & 2 \\
\hline & $4 b_{1}$ & 14.95 & 69.09 & 2 \\
\hline & $1 a_{2}$ & 14.40 & 65.23 & 2 \\
\hline & $6 a_{1}$ & $11.23^{\dagger}$ & 76.21 & 1 \\
\hline \multirow[t]{9}{*}{$\mathrm{N}_{2} \mathrm{O}$} & $1 \sigma$ & 561.71 & 794.52 & 2 \\
\hline & $2 \sigma$ & 431.00 & 601.81 & 2 \\
\hline & $3 \sigma$ & 426.85 & 602.05 & 2 \\
\hline & $4 \sigma$ & 44.49 & 72.53 & 2 \\
\hline & $5 \sigma$ & 39.55 & 73.81 & 2 \\
\hline & $6 \sigma$ & 22.41 & 77.25 & 2 \\
\hline & $1 \pi$ & 21.20 & 48.88 & 4 \\
\hline & $7 \sigma$ & 18.95 & 60.14 & 2 \\
\hline & $2 \pi$ & $12.89 *$ & 59.95 & 4 \\
\hline \multirow[t]{12}{*}{$\mathrm{O}_{3}$} & $1 a_{1}$ & 569.47 & 794.66 & 2 \\
\hline & $1 b_{1}$ & 563.07 & 794.48 & 2 \\
\hline & $2 \mathrm{a}_{1}$ & 563.07 & 794.50 & 2 \\
\hline & $3 a_{1}$ & 50.16 & 78.21 & 2 \\
\hline & $2 b_{1}$ & 40.63 & 80.62 & 2 \\
\hline & $4 a_{1}$ & 29.11 & 89.34 & 2 \\
\hline & $5 a_{1}$ & 23.66 & 69.13 & 2 \\
\hline & $1 b_{2}$ & 22.74 & 58.55 & 2 \\
\hline & $3 b_{1}$ & 22.36 & 77.09 & 2 \\
\hline & $4 b_{1}$ & 15.33 & 73.82 & 2 \\
\hline & $6 a_{1}$ & 14.65 & 76.56 & 2 \\
\hline & $1 a_{2}$ & $12.75^{\dagger}$ & 65.12 & 2 \\
\hline \multirow{11}{*}{$\begin{array}{l}\quad \mathrm{S}_{2} \text {, triplet } \\
\text { average of } \alpha \text { and } \beta \text { orbital values }\end{array}$} & $\sigma_{g} 2 \mathrm{~s}$ & 245.93 & 509.43 & 2 \\
\hline & $\sigma_{u}^{g} 2 \mathrm{~s}$ & 245.92 & 509.55 & 2 \\
\hline & $\pi_{u} 2 \mathrm{p}$ & 182.72 & 478.98 & 4 \\
\hline & $\pi_{g} 2 \mathrm{p}$ & 182.72 & 478.28 & 4 \\
\hline & $\sigma_{g} 2 \mathrm{p}$ & 182.81 & 478.98 & 2 \\
\hline & $\sigma_{u} 2 \mathrm{p}$ & 182.80 & 478.55 & 2 \\
\hline & $\sigma_{g} 3 \mathrm{~s}$ & 29.25 & $60.84 / 3$ & 2 \\
\hline & $\sigma_{u} 3 \mathrm{~s}$ & 22.35 & $72.54 / 3$ & 2 \\
\hline & $\sigma_{g} 3 \mathrm{p}$ & 13.42 & $54.39 / 3$ & 2 \\
\hline & $\pi_{u} 3 \mathrm{p}$ & 12.59 & $43.13 / 3$ & 4 \\
\hline & $\pi_{g} 3 \mathrm{p}$ & $9.36^{*}$ & $56.46 / 3$ & 2 \\
\hline \multirow[t]{15}{*}{$\mathrm{SO}_{2}$} & $1 b_{1}$ & 560.91 & 794.52 & 2 \\
\hline & $2 \mathrm{a}_{1}$ & 560.91 & 794.53 & 2 \\
\hline & $3 a_{1}$ & 249.31 & 509.66 & 2 \\
\hline & $2 b_{1}$ & 186.25 & 477.82 & 2 \\
\hline & $1 b_{2}$ & 186.18 & 477.98 & 2 \\
\hline & $4 a_{1}$ & 186.14 & 478.57 & 2 \\
\hline & $5 a_{1}$ & 41.53 & 76.54 & 2 \\
\hline & $3 b_{1}$ & 38.47 & 82.00 & 2 \\
\hline & $6 a_{1}$ & 23.49 & 81.17 & 2 \\
\hline & $7 a_{1}$ & 19.14 & 58.16 & 2 \\
\hline & $4 b_{1}$ & 19.06 & 74.31 & 2 \\
\hline & $2 b_{2}$ & 18.35 & 52.86 & 2 \\
\hline & $5 b_{1}$ & 14.65 & 64.71 & 2 \\
\hline & $1 \mathrm{a}_{2}$ & 14.10 & 59.21 & 2 \\
\hline & $8 a_{1}(S 3 p)$ & $12.50^{\dagger}$ & $66.21 / 3$ & 2 \\
\hline
\end{tabular}

values of $B$ are often smaller than theoretical ones, the BEB cross sections obtained using experimental $B$ values are usually higher (by $10 \%-15 \%$ at the cross section peak) than those obtained using theoretical $B$ values. Using the experimental value for the lowest electron binding energy (=first IP) will not only assure that the cross section starts at the right threshold but also we found that the shape and magnitude of the BEB cross section near the threshold agree better with known experimental cross sections. On the other hand, the cross sections with theoretical values of $B$ tend to agree better with experiment near the peak $(T \sim 100 \mathrm{eV})$.

For closed-shell molecules, we used the restricted Hartree-Fock (RHF) method with a Gaussian basis set (known as the 6-311-G set) provided by the GAMESS code. For open-shell molecules, we found that the unrestricted Hartree-Fock (UHF) method produced more realistic orbital energies-which we took as the electron binding energies as prescribed by the Koopman theorem-for valence orbitals than the restricted open-shell Hartree-Fock (ROHF) method. Although the UHF method has the disadvantage of producing a pair of orbital and kinetic energies ( $\alpha$ and $\beta$ orbitals) the valence orbital energies from the ROHF method were often unrealistically small, making the corresponding cross section too large. On the other hand, using $B$ and $U$ values from the UHF method produces cross sections almost identical to those produced using the average between the $B$ and $U$ values from the matching $\alpha$ and $\beta$ orbitals. The $B$ and $U$ values presented in Table II are these average values for open-shell molecules.

In practice, the BEB model is insensitive to minor variations in the values of $B$ and $U$ used, except for the lowest $B$. For this reason, we used the experimental values for the lowest $B$, which are available for many molecules, ${ }^{8}$ and theoretical values for the remaining orbitals. For example, the theoretical value for the lowest $B$ of CS is $12.68 \mathrm{eV}$ compared to the experimental value of $11.33 \mathrm{eV}$.

Using all experimental values of $B$ is not only difficult because often experimental data for inner shells do not exist, but also it is not necessarily more useful because there is no matching "experimental" values of $U$. Hence, we limited the use of experimental $B$ values to the first IPs only. Even then, we must decide whether to use the adiabatic IP or the vertical IP. Although photoionization and electron-impact ionization are not equivalent, the use of vertical IPs obtained from photoelectron analyses seems reasonable because such IPs are identified with the orbitals of the parent molecule, ${ }^{9}$ as our $B$ values are in the BEB model. Moreover, we found that, for molecules with significant differences between adiabatic and vertical IPs, the use of vertical IPs produced BEB cross sections in much better agreement with available experiments as is shown in Sec. III.

\section{APPLICATION TO MOLECULES}

In this section, we compare the BEB cross sections to the experimental values of ten molecules. We were unable to find experimental cross sections to be compared to the BEB cross section for $\mathrm{NO}_{2}$. Some experiments measured the 


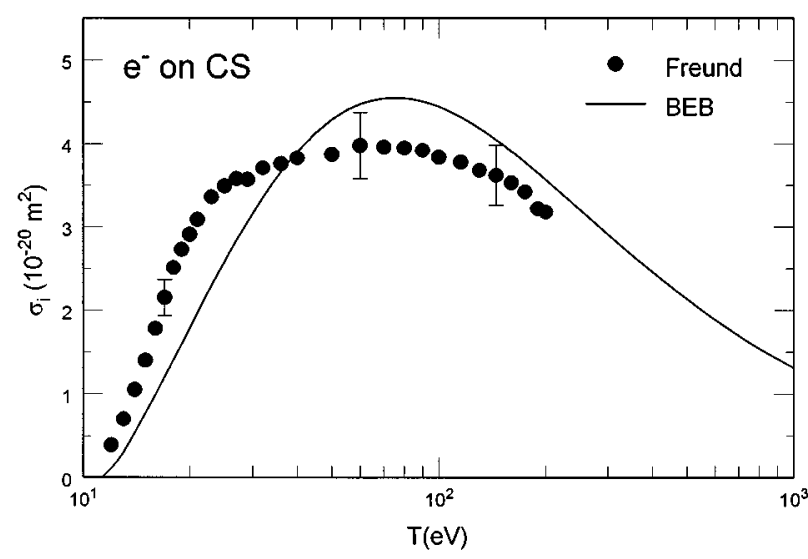

FIG. 1. Comparison of the BEB cross section to experiment for CS. Solid curve, the present work; circles, experimental data by Freund et al. (Ref. $10)$.

"gross" ionization cross section, which is determined by measuring the total ion current rather than the number of ions. On the other hand, BEB cross sections are "counting", ionization cross sections, which account for the number of singly charged ions produced. When many multiply charged ions are produced, a gross ionization cross section will be noticeably larger than a counting ionization cross section at high $T$. Hence, BEB cross sections should be considered as the lower limits to experimental gross ionization cross sections. In many experiments, both molecular ions as well as their fragments are collected using mass spectrometers. Since the BEB cross sections are simple sums of cross sections for ejecting one electron from each molecular orbital, the theory cannot give a detailed account of dissociative ionization or fragments produced. Hence, comparisons of the theory with experiments on large molecules with diverse channels for dissociative ionization and fragmentation are not straightforward. For simplicity, we compared our theoretical cross sections to the simple sum of all experimental partial cross sections that produced an ion. Nevertheless, the comparisons presented here will clearly demonstrate wide applicability of our theory.

\section{A. Carbon monosulfide (CS)}

Experimental data for CS by Freund et al. ${ }^{10}$ are compared to the BEB cross section in Fig. 1. The experimental data are partial cross sections for the production of $\mathrm{CS}^{+}$ions only, and hence should be lower than our theoretical cross section. The experimental data by Freund et al. show a general trend of being much higher than the BEB cross section for $T<30 \mathrm{eV}$ (see Figs. 1, 2, and 10) and then flattening for higher $T$ when the production of other fragmented ions becomes significant. The reason for the disagreement with the data by Freund et al. at low $T$ is unclear because the BEB cross sections for other molecules in this article and in Ref. 1 at low $T$ agree very well with experiment in shape and magnitude.

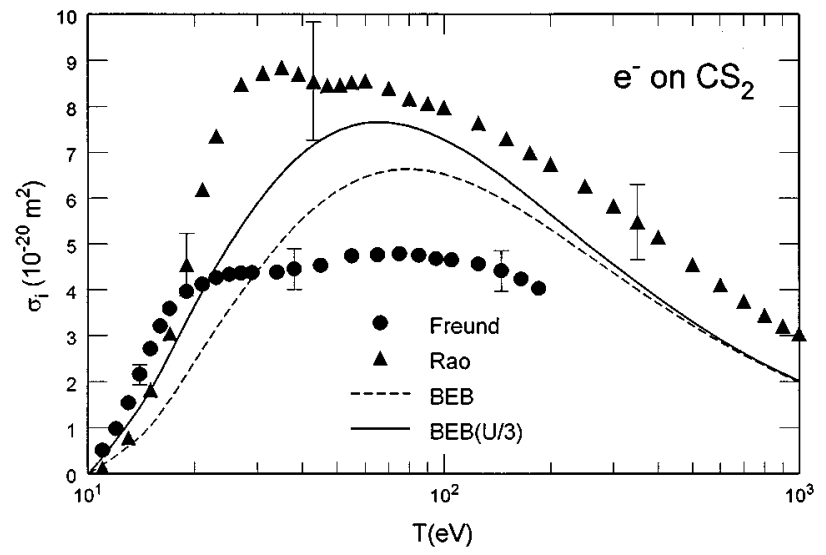

FIG. 2. Comparison of the BEB cross section to experiment for $\mathrm{CS}_{2}$. Solid curve, $\mathrm{BEB}$ cross section with a reduced value of $U^{\prime}=U / 3$ for the outermost molecular orbital; dashed curve, BEB cross section with the original $U$ values; circles, experimental data by Freund et al. (Ref. 10); triangles, experimental data by Rao and Srivastava (Ref. 11).

\section{B. Carbon disulfide $\left(\mathrm{CS}_{2}\right)$}

Experimental data for $\mathrm{CS}_{2}$ by Freund et al. ${ }^{10}$ and those by Rao and Srivastava ${ }^{11}$ are compared to the BEB cross section in Fig. 2. We also modified the BEB cross section in this case to use a reduced value of $U$ for the outermost valence molecular orbital, $2 \pi_{g}$, as we did in Ref. 1 . When a molecular orbital is dominated by an atomic orbital-in this case by the $3 p$ orbital of $\mathrm{S}$-with high principal quantum numbers, its kinetic energy is high, and makes the cross section too low because $U$ enters the cross section formula, Eq. (2), in the denominator. To correct this situation, we divide $U$ by the principal quantum number of the dominant atomic orbital. We used the Mulliken population analysis as our guide: when more than $90 \%$ of the population density of a molecular orbital comes from a valence atomic orbital, we used this reduction of $U$. The BEB cross section curve marked " $\mathrm{BEB}(\mathrm{U} / 3)$ " in the figure used the reduced value of $U$, as indicated in Table I.

As was the case for CS, the data by Freund et al. ${ }^{10}$ is a partial cross section for the production of $\mathrm{CS}_{2}^{+}$and should be a lower limit to the BEB cross section.

\section{Carbonyl sulfide (COS)}

The only set of experimental data for COS is the data by Srivastava, ${ }^{12}$ which is compared with the BEB cross section in Fig. 3. There is excellent agreement between theory and experiment for low $T<100 \mathrm{eV}$, but the experiment peaks at a higher $T$ than the theory. Also, the shape of the experimental cross section at $T>500 \mathrm{eV}$ disagrees with the theoretical shape. This trend at high $T$ is also seen in the experimental data on $\mathrm{H}_{2} \mathrm{~S}$ by the same group (see Fig. 5).

\section{Methane $\left(\mathrm{CH}_{4}\right)$}

We have published the BEB cross section for $\mathrm{CH}_{4}$ earlier ${ }^{1}$ using the adiabatic IP of $12.51 \mathrm{eV}{ }^{8}$ Methane is a typical example of a molecule affected by the Jahn-Teller 


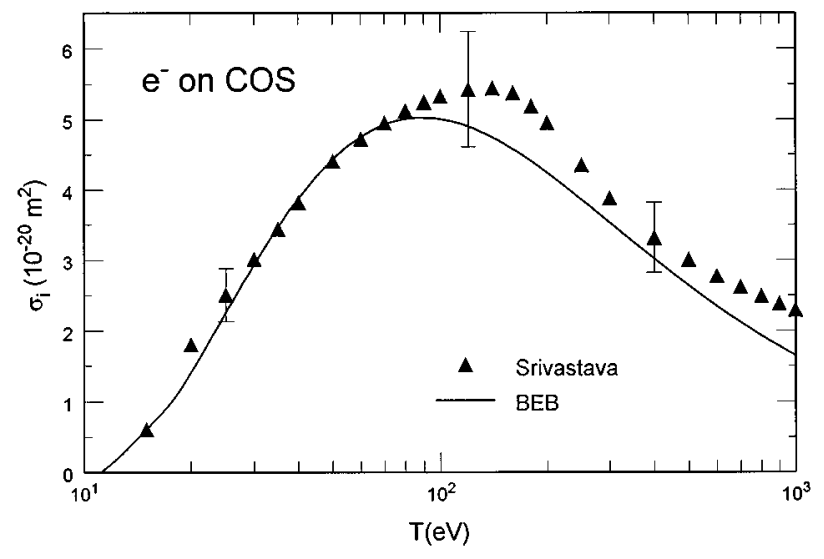

FIG. 3. Comparison of the BEB cross section to experiment for COS. Solid curve, the present work; triangles, experimental data by Srivastava (Ref. 12).

effect such that the photoionization peak of the six electrons in the $1 t_{2}$ valence orbital is spread out between 12.5 and 16 $\mathrm{eV} .{ }^{13}$ The center of gravity of this broad peak is approximately $14.25 \mathrm{eV}$, almost $2 \mathrm{eV}$ higher than the adiabatic IP. Because of the manner in which $B$ enters the BEB cross section through $S$ in Eq. (2), a higher $B$ value results in a smaller cross section. In Fig. 4, we compare available experimental data ${ }^{14-17}$ with the BEB cross sections using the adiabatic and vertical IPs. It is clear that the agreement between theory and experiment between the threshold and $T<100 \mathrm{eV}$ is significantly improved by using the vertical IP.

\section{E. Hydrogen sulfide $\left(\mathrm{H}_{2} \mathrm{~S}\right)$}

Experimental data for $\mathrm{H}_{2} \mathrm{~S}$ by Rao and Srivastava ${ }^{18}$ and those by Belić and Kurepa ${ }^{19}$ are compared to the BEB cross section in Fig. 5. The data by Rao and Srivastava agree very well with the BEB cross section which used a reduced value of $U$ for the outermost molecular orbital, $2 \mathrm{~b}_{2}$. The experi-

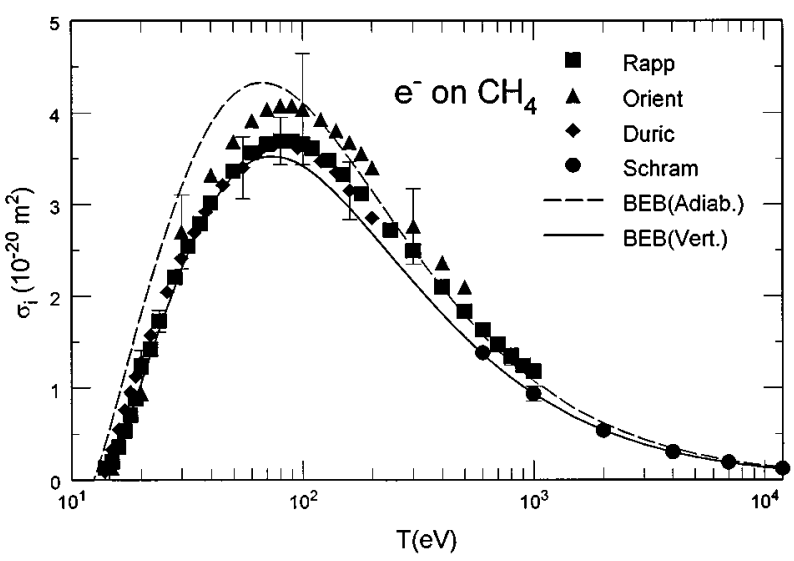

FIG. 4. Comparison of the BEB cross section to experiment for $\mathrm{CH}_{4}$. Solid curve, the present work with the vertical IP; dashed curve, BEB cross section with the adiabatic IP (Ref. 1); squares, experimental data by Rapp and Englander-Golden (Ref. 14); triangles, experimental data by Orient and Srivastava (Ref. 15); diamonds, experimental data by Durić et al. (Ref. 16); circles, experimental data by Schram et al. (Ref. 17).

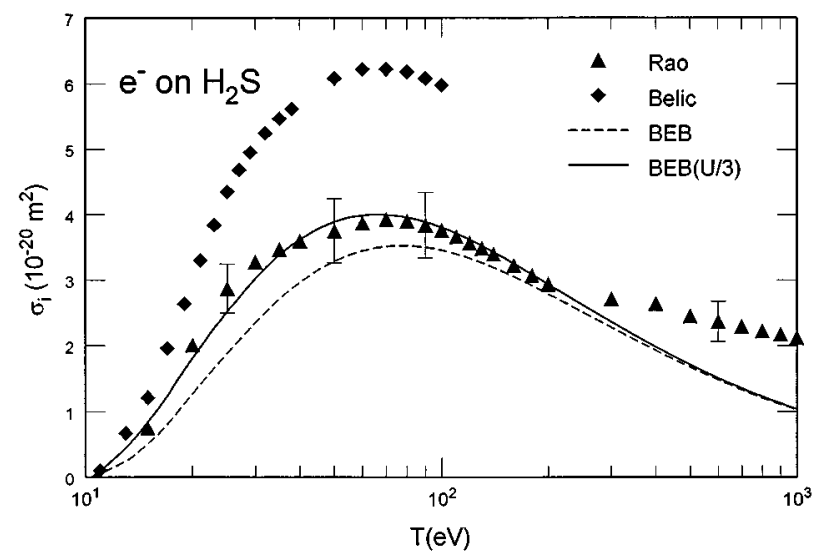

FIG. 5. Comparison of the BEB cross section to experiment for $\mathrm{H}_{2} \mathrm{~S}$. Solid curve, BEB cross section with a reduced value of $U^{\prime}=U / 3$ for the outermost molecular orbital; dashed curve, BEB cross section with the original $U$ values; diamonds, experimental data by Belić and Kurepa (Ref. 19); triangles, experimental data by Rao and Srivastava (Ref. 18).

mental data, however, deviates from the theory for $T>300$ $\mathrm{eV}$ in the same manner as the experimental data ${ }^{12}$ for COS (see Fig. 3). We found that the data by Belić and Kurepa agree with our theory very well when we renormalized their peak value to the theory. The renormalized data became so close to those by Rao and Srivastava that it was difficult to distinguish the two sets of data.

Khare and Meath ${ }^{20}$ used a method similar to ours in spirit, i.e., a combination of the Mott cross section and the Bethe theory. Their theory uses empirical values of $d f / d W$ and provides partial ionization cross sections. However, the sum of their partial cross sections for $\mathrm{H}_{2} \mathrm{~S}$ is about one-half of the BEB cross section.

\section{F. Ammonia $\left(\mathrm{NH}_{3}\right)$}

Similar to the case of $\mathrm{CH}_{4}$, the BEB cross section we have published earlier ${ }^{1}$ with the adiabatic IP was too high at $T<100 \mathrm{eV}$ compared to available experiments. ${ }^{21,22}$ The neutral $\mathrm{NH}_{3}$ molecule is pyramidal while the $\mathrm{NH}_{3}^{+}$ion is planar. This change in shape makes the vertical IP to be $10.88 \mathrm{eV}^{23}$ while the adiabatic IP is $10.16 \mathrm{~V} .^{8}$ Although this difference is not as pronounced as the difference in $\mathrm{CH}_{4}$, we still found the use of the vertical IP improved the agreement between experiments and the BEB cross section at $T<100 \mathrm{eV}$ as is shown in Fig. 6.

\section{G. Nitrogen dioxide $\left(\mathrm{NO}_{2}\right)$}

To the best of our knowledge, there is no experimental data for the total ionization cross section of $\mathrm{NO}_{2}$ to compare to the BEB cross section in Fig. 7. Unlike other molecules in this article, $\mathrm{NO}_{2}$ is an open-shell molecule, and we used the UHF method to calculate the ground-state wave function. The value of $B$ and $U$ are the average of those for $\alpha$ and $\beta$ orbitals as was mentioned in Sec. II. Also, $\mathrm{NO}_{2}$ is an 


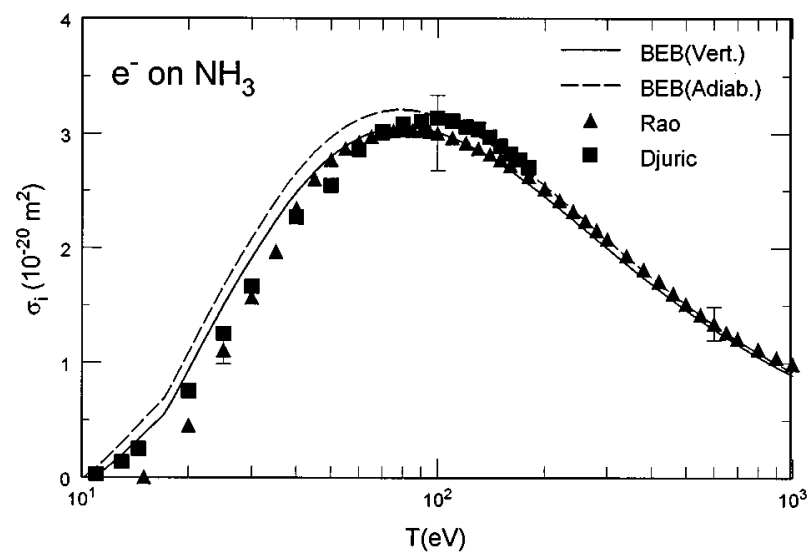

FIG. 6. Comparison of the BEB cross section to experiment for $\mathrm{NH}_{3}$. Solid curve, present work with the vertical IP; dashed curve, BEB cross section with the adiabatic IP (Ref. 1); triangles, experimental data by Rao and Srivastava (Ref. 22); squares, experimental data by Durić et al. (Ref. 21).

unusual molecule in that more $\mathrm{NO}^{+}$ions are produced than $\mathrm{NO}_{2}^{+}$ions at low $T$ both by electron-impact ionization ${ }^{24}$ and by photoionization. ${ }^{25}$

Similar to $\mathrm{NH}_{3}, \mathrm{NO}_{2}$ changes its shape upon ionization: The neutral $\mathrm{NO}_{2}$ is bent while the $\mathrm{NO}_{2}^{+}$ion is linear. The vertical IP is $11.23 \mathrm{eV}^{9}$ while the adiabatic IP is $9.75 \mathrm{eV}^{8}$ This difference is large enough to make the BEB cross section at $T<100 \mathrm{eV}$ to reduce visibly in Fig. 7 .

\section{H. Nitrous oxide $\left(\mathrm{N}_{2} \mathrm{O}\right)$}

Two sets of experimental data for $\mathrm{N}_{2} \mathrm{O}$, those by Rapp and Englander-Golden ${ }^{14}$ and those by Iga et al., ${ }^{26}$ are compared to the BEB cross section in Fig. 8. Both sets of data and the theory in Fig. 8 are in excellent agreement with each other. The data by Rapp and Englander-Golden are a classic example of a gross ionization cross section, where the ion current was measured instead of counting the number of ions produced. Of the many molecules we have applied the BEB theory so far in Refs. 1 and 2 and the present work, the experimental data by Rapp and Englander-Golden consis-

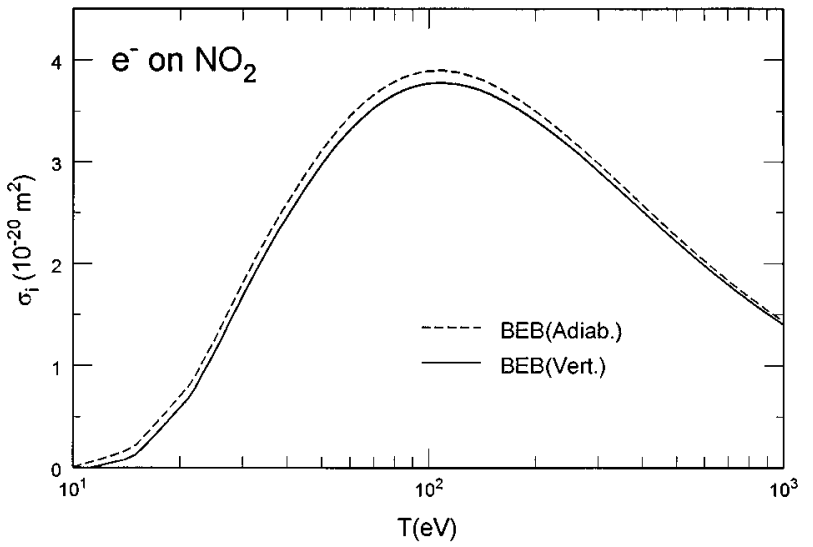

FIG. 7. BEB cross section for $\mathrm{NO}_{2}$. Solid curve, present work with the vertical IP; dashed curve, present work with the adiabatic IP.

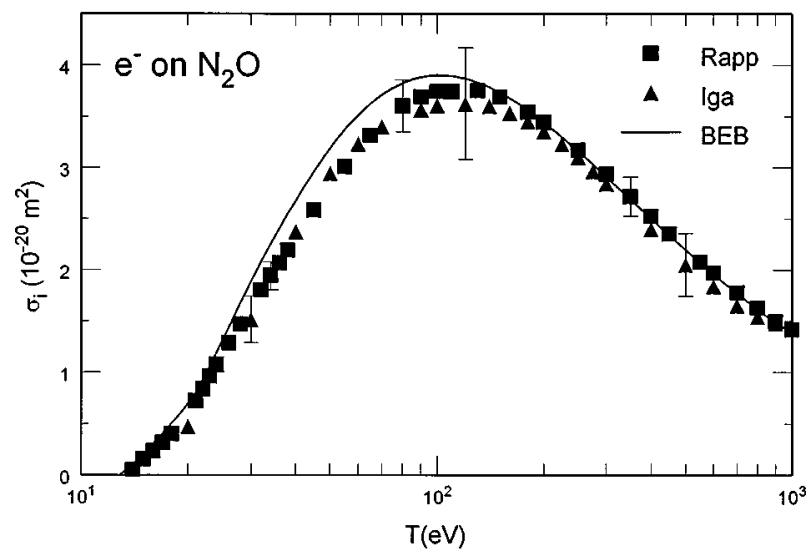

FIG. 8. Comparison of the BEB cross section to experiment for $\mathrm{N}_{2} \mathrm{O}$. Solid curve, the present work; squares, experimental data by Rapp and EnglanderGolden (Ref. 14); triangles, experimental data by Iga et al. (Ref. 26).

tently agree with the theory within $15 \%$ and often much better, making us believe that the BEB model is very reliable for total ionization cross sections.

Märk et al. ${ }^{27}$ measured partial ionization cross section of $\mathrm{N}_{2} \mathrm{O}$ for the production of $\mathrm{N}_{2} \mathrm{O}^{+}$by electron impact. Their peak cross section is $1.49 \times 10^{-20} \mathrm{~m}^{2}$ at $T=110 \mathrm{eV}$.

\section{Ozone $\left(\mathrm{O}_{3}\right)$}

Experimental data by Siegel $^{28}$ and those by Newson et $a .^{29}$ on $\mathrm{O}_{3}$ are compared to the BEB cross section in Fig. 9. Siegel's data are absolute, while Newson et al. normalized to the Siegel data. Although Newson et al. identifies their data as partial cross sections, they have cross sections for the production of $\mathrm{O}_{3}^{+}, \mathrm{O}_{2}^{+}$, and $\mathrm{O}^{+}$. Their data in Fig. 9 are the sum of these three sets of partial cross sections, and hence can be regarded as the total ionization cross section.

When we renormalized the data by Newson et al. to match the theoretical peak value, their experimental data and the $\mathrm{BEB}$ cross section agreed very well for the entire range

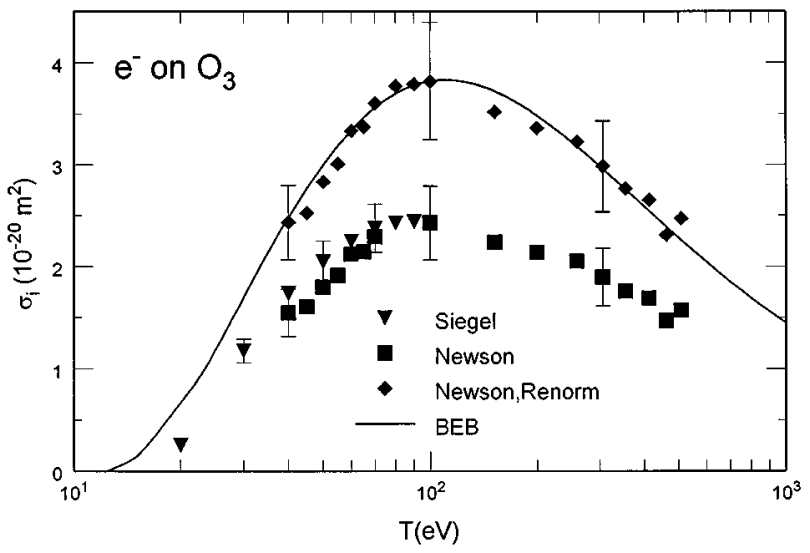

FIG. 9. Comparison of the BEB cross section to experiment for $\mathrm{O}_{3}$. Solid curve, the present work; triangles, experimental data by Siegel (Ref. 28); squares, experimental data by Newson et al. (Ref. 29); diamonds, renormalized data by Newson $e t$ al. 


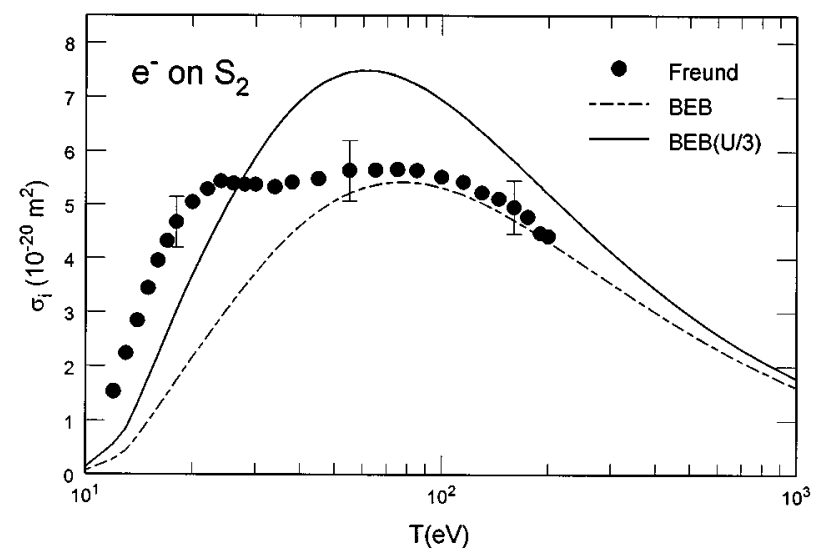

FIG. 10. Comparison of the BEB cross section to experiment for $\mathrm{S}_{2}$. Solid curve, $\mathrm{BEB}$ cross section with reduced values of $U^{\prime}=U / 3$ for the valence molecular orbitals; dashed curve, BEB cross section with the original $U$ values; circles, experimental data by Freund et al. (Ref. 10).

of $T$ as can be seen in Fig. 9. This is an indication that the Siegel data may have been too low, perhaps by about $60 \%$. The difference between the vertical IP of $12.75 \mathrm{eV}^{9}$ and the adiabatic IP of $12.43 \mathrm{eV}^{8}$ is too small to make any discernible difference in the BEB cross section.

\section{J. Disulfur $\left(\mathbf{S}_{2}\right)$}

The experimental data for $\mathrm{S}_{2}$ by Freund et al. ${ }^{10}$ are compared to the BEB cross section in Fig. 10. The ground state of $\mathrm{S}_{2}$ is an open-shell, triplet state. Hence, we used the UHF method, and averaged the $B$ and $U$ values for the $\alpha$ and $\beta$ molecular orbitals. Since $S_{2}$ is a homonuclear diatomic molecule, the atomic orbitals from each atom equally contribute to a matching molecular orbital.

We now face the question of whether or not to divide the $U$ values of the outer molecular orbitals by the principal quantum number of the matching atomic orbital, as we did in some molecules with atoms that contain M-shell atomic orbitals. We chose to do this when a molecular orbital was dominated by an atomic orbital with a high $u=U / B$ value to bring the BEB cross section into a better agreement with experiment. Comparison with the experimental data by Freund et al. (see Fig. 10) indicates that we should use reduced values of $U$ because the experimental data are partial cross sections corresponding to the production of $\mathrm{S}_{2}^{+}$only, and should lie below our BEB cross section at high $T$. The flat shape of the experimental data in Fig. 10 above $T=20 \mathrm{eV}$ is an indication of missing ions other than the parent ion $\mathrm{S}_{2}^{+}$.

\section{K. Sulfur dioxide $\left(\mathrm{SO}_{2}\right)$}

Unlike $\mathrm{NO}_{2}$, there are many sets of experimental data for $\mathrm{SO}_{2} \cdot{ }^{30-33}$ We compare them to the BEB cross section in Fig. 11. Basner et al. ${ }^{32}$ actually report three distinct sets of cross sections, one set measured at the Universität Greifswald in Germany, another set measured at the City University of New York, and a semiempirical cross section based on an additivity rule by one of the authors at Greifswald. We

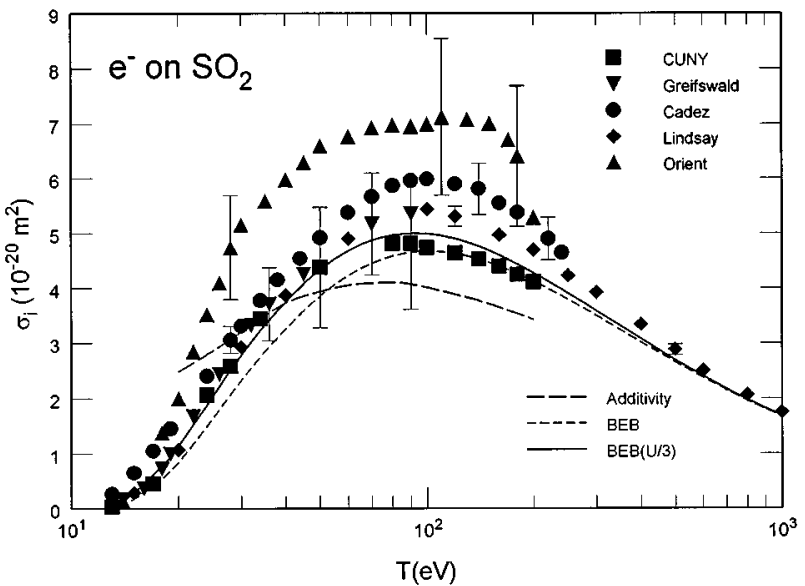

FIG. 11. Comparison of the BEB cross section to experiment for $\mathrm{SO}_{2}$. Solid curve, $\mathrm{BEB}$ cross section with a reduced value of $U^{\prime}=U / 3$ for the valence molecular orbital; medium-dashed curve, BEB cross section; long-dashed curve, semiempirical additivity rule (Ref. 32); circles, experimental data by Cadež et al. (Ref. 30); triangles, experimental data by Orient and Srivastava (Ref. 31); squares, experimental data by the CUNY group (Ref. 32); inverted triangles, experimental data by the Greifswald group (Ref. 32); diamonds, experimental data by Lindsay et al. (Ref. 33).

present these three sets of cross sections individually in Fig. 11, and except for additivity, they are all in good agreement with the BEB cross section with a reduced value of $U^{\prime}=U / 3$. The BEB cross section with $U^{\prime}$ also agrees with the latest experimental data by Lindsay et al., ${ }^{33}$ though not by the $\pm 3.5 \%$ accuracy claimed by the authors. It is clear from Fig. 11 that the BEB cross section is reliable within $\sim 10 \%$ from the threshold to $T=1 \mathrm{keV}$.

The outermost molecular orbital, $8 \mathrm{a}_{1}$, is dominated by the 3 p atomic orbital of sulfur, and hence we divided its $U$ value by 3 as is shown in Table II. Again, the difference between the vertical IP of $12.50 \mathrm{eV}^{9}$ and the adiabatic IP of $12.32 \mathrm{eV}^{8}$ is too small to affect the BEB cross section.

\section{CONCLUSION}

We have demonstrated that BEB cross sections provide reliable electron-impact total ionization cross sections for a large variety of molecules, from the ionization threshold to incident energies in the $\mathrm{keV}$ range. The calculation of BEB cross sections requires only a minimal set of constants from molecular orbitals for the ground state of the target molecule, which are readily available from molecular structure codes.

We have also shown that the use of the vertical IP rather than the adiabatic IP significantly improves the agreement between theory and experiment at $T<100 \mathrm{eV}$ when the vertical IP is larger than the adiabatic IP by $\sim 1 \mathrm{eV}$ or more. Although there are references on vertical IPs of common molecules, we would like to see more photoelectron data on vertical IPs of radicals and molecules of interest to plasma chemistry and semiconductor fabrication. One can usually judge if a vertical IP will be sufficiently different from the matching adiabatic IP by comparing the geometrical shape of the parent molecule and that of the resulting molecular ion. 
Molecules with strong Jahn-Teller effect are also expected to show significant differences between vertical and adiabatic IPs.

Moreover, the BEB equation consists of a simple analytic function of the incident energy for each molecular orbital that contributes to the ionization cross section, making the equation ideally suited for modeling applications. Molecular orbital constants needed to construct BEB cross sections for the molecules in this article are presented in Tables I and II.

Work is in progress to extend the BEB model to other molecules of interest to plasma chemistry modeling.

\section{ACKNOWLEDGMENTS}

We are grateful to the creators of the GAMESS code (see Ref. 7), without which we could not have carried out this work. We thank S. K. Srivastava, H. C. Straub, T. M. Märk, and $\mathrm{K}$. Becker for providing their experimental data, some of them prior to publication. One of us (Y.K.K.) is indebted to J. Berkowitz for his help in identifying references on vertical IPs. This work at NIST was supported in part by the Office of Fusion Energy of the U.S. Department of Energy, and at the University of Nebraska-Lincoln by the National Science Foundation Grant No. PHY-9119818.

${ }^{1}$ W. Hwang, Y.-K. Kim, and M. E. Rudd, J. Chem. Phys. 104, 2956 (1996).

${ }^{2}$ Y.-K. Kim and M. E. Rudd, Phys. Rev. A 50, 3954 (1994).

${ }^{3}$ M. L. Marconi, D. A. Mendis, A. Korth, R. P. Lin, D. L. Mitchell, and H. Reme, Astrophys. J. 352, L17 (1990).

${ }^{4}$ L. Vriens, in Case Studies in Atomic Physics, Vol. 1, edited by E. W. McDaniel and M. R. C. McDowell (North Holland, Amsterdam, 1969), p. 335.

${ }^{5}$ H. Bethe, Ann. Phys. 5, 325 (1930).

${ }^{6}$ N. F. Mott, Proc. R. Soc. London, Ser. A 126, 259 (1930).

${ }^{7}$ We have used the version of GAMESS described by M. W. Schmidt, K. K. Baldridge, J. A. Boatz, S. T. Elbert, M. S. Gordon, J. H. Jensen, S. Koseki, N. Matsunaga, K. A. Nguyen, S. J. Su, T. L. Windus, M. Dupuis, and J. A. Montgomery, J. Comput. Chem. 14, 1347 (1993).

${ }^{8}$ S. G. Lias, J. F. Liebman, R. D. Levin, and S. A. Kafafi, "NIST Positive
Ion Energetics Database, Version 2.0," Standard Reference Database 19A, National Institute of Standards and Technology, Oct. 1993. This database lists adiabatic IPs.

${ }^{9}$ K. Kimura, S. Katsumata, Y. Achiba, T. Yamazaki, and S. Iwata, Handbook of He I Photoelectron Spectra of Fundamental Organic Molecules (Japan Scientific Soc., Tokyo, 1981); K. Kimura, Photoelectron Spectra of Unstable Molecules (Inst. for Molecular Science, 1984). These publications list vertical IPs.

${ }^{10}$ R. S. Freund, R. C. Wetzel, and R. J. Shul, Phys. Rev. A 41, 5861 (1990).

${ }^{11}$ M. V. V. S. Rao and S. K. Srivastava, J. Geophys. Res. 96, 17563 (1991).

${ }^{12} \mathrm{~S}$. K. Srivastava (private communication).

${ }^{13}$ A. W. Potts and W. C. Price, Proc. R. Soc. London, Ser. A 326, 165 (1972).

${ }^{14}$ D. Rapp and P. Englander-Golden, J. Chem. Phys. 43, 1464 (1965).

${ }^{15}$ O. J. Orient and S. K. Srivastava, J. Phys. B 20, 3923 (1987).

${ }^{16}$ N. Durić, I. Čadež, and M. Kurepa, Int. J. Mass. Spectrom. Ion Processes 108, R1 (1991).

${ }^{17}$ B. L. Schram, M. J. van der Wiel, F. J. de Heer, and H. R. Moustafa J. Chem. Phys. 44, 49 (1966).

${ }^{18}$ M. V. V. S. Rao and S. K. Srivastava, J. Geophys. Res. 98, 13137 (1993).

${ }^{19}$ D. S. Belić and M. V. Kurepa, Fizika 17, 117 (1985).

${ }^{20}$ S. P. Khare and W. J. Meath, J. Phys. B 20, 2101 (1987).

${ }^{21}$ N. Djurić, D. Belić, M. Kurepa, J. U. Mack, J. Rothleitner, and T. D. Märk, Abstracts, 12th Int. Conf. on the Physics of Atomic and Electronic Collisions, edited by S. Datz (Gatlinburg, 1981), p. 384.

${ }^{22}$ M. V. V. S. Rao and S. K. Srivastava, J. Phys. B 25, 2175 (1992).

${ }^{23}$ A. W. Potts and W. C. Price, Proc. R. Soc. London, Ser. A 326, 181 (1972).

${ }^{24}$ K. Stephan, H. Helm, Y. B. Kim, G. Seykora, J. Ramler, and M. Grössl, J. Chem. Phys. 73, 303 (1980).

${ }^{25}$ V. H. Dibeler, J. A. Walker, and S. K. Liston, J. Res. NBS A 71, 371 (1967)

${ }^{26}$ I. Iga, M. V. V. S. Rao, and S. K. Srivastava, J. Geophys. Res. 101, 9261 (1996).

${ }^{27}$ E. Märk, T. D. Märk, Y. B. Kim, and K. Stephan, J. Chem. Phys. 75, 4446 (1981).

${ }^{28}$ M. W. Siegel, Int. J. Mass Spectrom. Ion Phys. 44, 19 (1982).

${ }^{29}$ K. A. Newson, S. M. Lee, S. D. Price, and N. J. Mason, Int. J. Mass Spec. Ion Processes 148, 203 (1995).

${ }^{30}$ I. M. Čadež, P. M. Prejčev, and M. V. Kurepa, J. Phys. D 16, 305 (1983).

${ }^{31}$ O. J. Orient and S. K. Srivastava, J. Chem. Phys. 80, 140 (1984).

${ }^{32}$ R. Basner, M. Schmidt, H. Deutsch, V. Tarnovsky, A. Levin, and K. Becker, J. Chem. Phys. 103, 211 (1995).

${ }^{33}$ B. G. Lindsay, H. C. Straub, K. A. Smith, and R. F. Stebbings, J. Geophys. Res. 101, 21151 (1996). 